\title{
An observational study on clinical, epidemiological and laboratory profile in patients of dengue fever admitted to a tertiary care hospital in Kolkata, West Bengal, India in the year 2016
}

\begin{abstract}
Dengue fever is an important viral hemorrhagic fever amongst the arthropod-borne virus illnesses. Dengue suspect patients admitted in the School of Tropical Medicine, Kolkata after being screened for inclusion and exclusion criteria and taken as a case. Objective of the study was to evaluate the detailed clinical picture, laboratory parameters, complications of Dengue fever, its outcome and epidemiological profile. After detailed history, clinical examination and necessary investigations patients were managed according to NVBDCP guidelines. In this study, a total of 103 dengue IgM ELISA positive or Dengue NS1Ag ELISA positive or both patients were included and analyzed. Amongst clinical manifestations most of the patients had myalgia (85.4\%), followed by rash $(56.3 \%)$, vomiting $(37.9 \%)$ and arthralgia $(24.3 \%)$. Bleeding manifestations were noted in $37 \%$ of patients. $21 \%$ of the patients had plasma leakage. Only 13\% patients had hypotension which was corrected by only volume replacement in $9 \%$ patients. In LFT $85.43 \%$ patients showed raised SGOT, $67.86 \%$ showed raised SGPT and only minority $(1.9 \%)$ had raised bilirubin level. SGOT $>$ SGPT were present in $83 \%$ patients. In analysis of SGOT elevation 2-5-fold elevation was common $(62 \%)$. But in analysis of SGPT elevation 2-5-fold elevation and 1-2-fold elevation were comparable. In making a diagnosis $41 \%$ cases Dengue IgM was helpful followed by $35 \%$ for Dengue NS1Ag and only $1 / 5^{\text {th }}$ of patients had both tests reactive. Platelet values and days counted from the onset of fever were followed and no specific association was noted. $13.4 \%$ of patients had pancreatic involvement and all of them had remarkable/significant lipase elevation than amylase. In our study, some new clinic-biochemical parameters were noted in hepatic, pancreatic involvement, sero-diagnosis, bleeding patterns, platelet count trends and plasma leakage. These should always be kept in mind during an epidemic which would help in managing dengue fever and predicting its outcome.
\end{abstract}

Keywords: hemorrhagic fever, arthropod-borne, illnesses, mosquitoes, pain
Volume 5 Issue 4 - 2017

\author{
Bhattacharjee B, Bhattacharya S, Majumdar \\ D, Chakraborty S, Sardar B, Naskar A, Ghosh \\ MK, Mallik S, Saha B \\ Department of Tropical Medicine, India
}

Correspondence: Boudhayan Bhattacharjee, Department of Tropical Medicine, School of Tropical Medicine, 108, Chittaranjan Avenue, Kolkata, State- West Bengal, India, Tel 91-9477031946, Emailbou.bhatta@gmail.com

Received: July 25, 2017 | Published: August 23, 2017

\section{Introduction}

Dengue fever is an important viral hemorrhagic fever amongst the arthropod-borne virus illnesses. It is caused by arbo-virus of flavi virus genus with 4 serotypes. Its vectors are Aedes aegypti and Aedes albopictus mosquitoes. A clinical manifestation ranges from undifferentiated fever to florid hemorrhage and shock. It depends on age of the patient, virus strain and immune status of the host. Clinically dengue fever is described as an acute febrile illness of 2-7 days duration with two or more of the following symptoms headache, retro-orbital pain, myalgia, arthralgia, rash, hemorrhagic manifestations. ${ }^{1}$ Diagnosis of Dengue hemorrhagic fever is made when a case with clinical criteria of dengue fever presents with hemorrhagic tendencies evidenced by one or more of the following, a) positive tourniquet test b) petechiae, ecchymoses or purpura c) bleeding from mucosa, gastrointestinal tract, injection sites or other sites plus thrombocytopenia $(<100000$ cells per $\mathrm{cmm}$ ) plus evidence of plasma leakage due to increased vascular permeability, manifested by one or more of the following: 1) A rise in average hematocrit for age and sex $\geq 20 \%, 2$ ) A more than $20 \%$ drop in hematocrit following volume replacement treatment compared to baseline, 3) Signs of plasma leakage (pleural effusion, ascites, hypoproteinemia). ${ }^{1}$

Dengue shock syndrome is diagnosed when all the above criteria for DHF is met with evidence of circulatory failure manifested by rapid and weak pulse and narrow pulse pressure $(<20 \mathrm{mmHg})$ or hypotension for age, cold and clammy skin and restlessness. ${ }^{2}$ The first evidence of occurrence of DF in the country was reported during 1956 from Vellore district in Tamil Nadu. The first DHF outbreak occurred in Calcutta (West Bengal) in 1963 out of which $30 \%$ of cases showing hemorrhagic manifestations. All the four serotypes i.e. Dengue 1, 2, 3 and 4 have been isolated from India.$^{1}$. West Bengal has been experiencing the problem of dengue outbreak since long. Almost every year with the advent of rainy season from the month of July to November West Bengal faces either a simple or an extensive outbreak of dengue infection. Dengue Infection can present as a varied presentation starting from a simple fever with myalgia to a severe multi organ dysfunction syndrome involving brain, liver, kidney, lung, pancreas and heart. Hepatic involvement is one of the common presentations in dengue fever. Pattern of liver enzymes and bilirubin levels may be used as a prognostic marker .2. Thrombocytopenia (with or without bleeding manifestations), raised pancreatic enzymes, coagulopathy are seen often in severe dengue infection. During recent outbreaks in India, the clinical manifestations which were shown by the patients were slightly different from those in past years, more severe complications have been noticed in recent times. Dengue infection threatens people every year till today in West Bengal. ${ }^{3,4}$ Objective of our study was to evaluate the detailed clinical picture, laboratory parameters and complications of dengue fever, to 
detect epidemiological profile of dengue fever and to detect outcome of dengue in relation to clinical, laboratory picture and treatment provided.

\section{Material and methods}

\section{Study area}

The study was conducted in patients admitted to the Tropical Medicine department in School of Tropical Medicine, Kolkata, coming from different parts of West Bengal, India.

\section{Study population}

The study population comprised of newly diagnosed adults and children who were admitted at the Tropical Medicine department of School of Tropical Medicine, Kolkata.

\section{Study design}

This was a cross-sectional, observational study.

\section{Study duration}

From May 2016 to December, 2016.

\section{Inclusion criteria}

Confirmed Dengue patients (By Dengue NS1Ag or Dengue IgM or both, tests are done by ELISA method) who were willing to participate in the study (consent being given by the patient or a close relative or guardian in case of a minor).

\section{Exclusion criteria}

a. Those who were unwilling to participate in the study.

b. Patients with concurrent bacterial/viral/parasitic infection.

c. Patients with chronic liver disease, chronic kidney disease, heart disease or any other chronic diseases.

\section{Study technique}

Dengue suspect patients admitted in the IPD were screened for inclusion and exclusion criteria and were taken as a case. Thorough history and clinical examination was done and were recorded. Blood samples on the first day of observation were taken for routine complete hemogram, liver function test and lipid profile, renal function tests which will be done from Department of Biochemistry and Laboratory Medicine, STM. Complete hemogram was done on daily basis till discharge to follow trend of thrombocytopenia. X-Ray chest and ultra-sonography of whole abdomen, Electrocardiogram was done to rule out any pleural effusion, ascites or GB edema and any preexisting condition. Other investigations were done as the clinical condition demands. Details of treatment received and outcome were recorded. All these data were tabulated in a proforma and analyzed. Patients were managed and followed up as per National Vector Borne Disease Control Program guideline in the Inpatients department as per patient's clinical condition.

\section{Tool of study:}

a. Pre-designed and Pre-tested schedule, with proper history, clinical examination and all blood reports including dengue virus serology.

b. Automated analyzer for pathological and biochemical tests.

c. Machine for Dengue Final serology (by ELISA). d. X-ray chest.

e. Ultra-sonography who fulfilled the inclusion criteria and consent to participating in the study was included.

Sample size: 103 .

Statistical analysis: Data collected was compiled and analyzed using appropriate statistical methods.

Ethics: The study protocol was submitted to the Clinical Research Ethics Committee of Calcutta School of Tropical Medicine, Kolkata and was reviewed and approved.

Patient information and informed consent: Informed consent process was undertaken in accordance with the latest ICMR Guidelines. Risks, if any and benefits of study participation were explained to the patient during the administration of informed consent process. Written consent was obtained from the patient and/or legal representative. In case of a patient who cannot provide informed consent in writing a left thumb print to indicate consent in the presence of at least one witness was acceptable.

\section{Results}

In this study, total 103 dengue MAC ELISA positive or Dengue NS1Ag ELISA positive (Tested as per day of fever according to NVBDCP guidelines) or both patients were included and analyzed. If the patient presented within 5 days of fever NS1 Ag was tested and irrespective of results Dengue IgM was tested after 5 days of fever during treatment. But those who presented late only Dengue IgM was tested. Among a total of 103 patients, 61(59\%) were male and 42(41\%) female (Figure 1). Mean age of the patients was $32.02 \pm 14.875$ (Figure $2)$. Most of the patients were from urban $(85 \%)$ and the remainder from rural areas and semi-urban areas (Figure 3). Amongst clinical manifestations most of the patients had myalgia (85.4\%), followed by rash $(56.3 \%)$ and vomiting (37.9\%). However only $24.3 \%$ of patients had arthralgia (Figure 4). Gastrointestinal symptoms were present in the form of diarrhea and abdominal pain $9.7 \%$ each (Figure 4). In 37\% of patients there were bleeding manifestations (Figure 5). Patients with bleeding manifestations purpuric spots was in the majority $(10 \%)$, hematuria, gum bleeding were $6 \%$ each, PV bleeding was 8 $\%$ and sub-conjunctival hemorrhage was $5 \%$ (Figure 5). $21 \%$ of the patients had plasma leakage (Figure 6). And amongst who had leakage were $8 \%$ for pleural effusion, $5 \%$ were having ascites and $8 \%$ were having both pleural effusion and ascites (Figure 6). Only 13\% patients had hypotension which was corrected by only volume replacement in $9 \%$ patients (Figure 7). $40 \%$ of patients had organomegaly and hepatomegaly amongst them $27 \%$ had hepatomegaly and only small proportion $(2.9 \%)$ had lymphadenopathy (Figure 8$)$. In Liver function tests $85.43 \%$ patients had raised ( $>1 \mathrm{xULN}$ ) SGOT, $67.86 \%$ had raised SGPT ( $>1 \times$ ULN) and only minority $(1.9 \%)$ had raised bilirubin level (more than $1 \mathrm{mg} / \mathrm{dl}$ ) (Figure 9). SGOT $>$ SGPT were present in $86(83 \%)$ patients (Figure 10). In analysis of SGOT 2-5-fold elevation was common (62\%), 1-2-fold elevation was only $16 \%$ (Figure 11 ). But in analysis of SGPT 2-5-fold elevation was 45\% and 1-2-fold elevation was $35 \%$ (Figure 12). In the study population $42(41 \%)$ patients were Dengue IgM reactive, 24 (23\%) patients were Dengue NS1 Ag reactive and $36(35 \%)$ patients were both reactive (Figure 13). Only $11 \%$ patients required platelet transfusion (Figure 14). Amongst them gum bleeding was the most common (46\%) indication followed by PV bleeding $(27 \%)$ and for low platelet count $(<10,000 / \mathrm{cm}) 18 \%$ were transfused (Figure 14). Platelet values and days counted from the onset of fever were followed. But at which day of fever platelet count falls and when it rises has no specific pattern (Figure 15). Only 
14 patients (13.4\%) had pancreatic involvement (Figure 16) in course of the illness and lipase elevation ( $>4 \mathrm{x}$ ULN) was more than amylase in all cases (Figure $17 \& 18$ ).

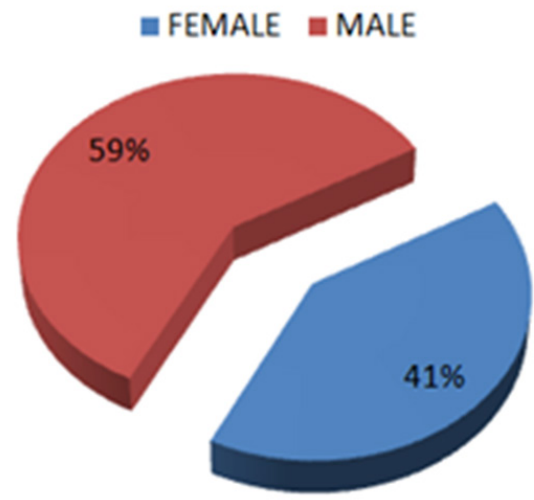

Figure I Sex Distribution.

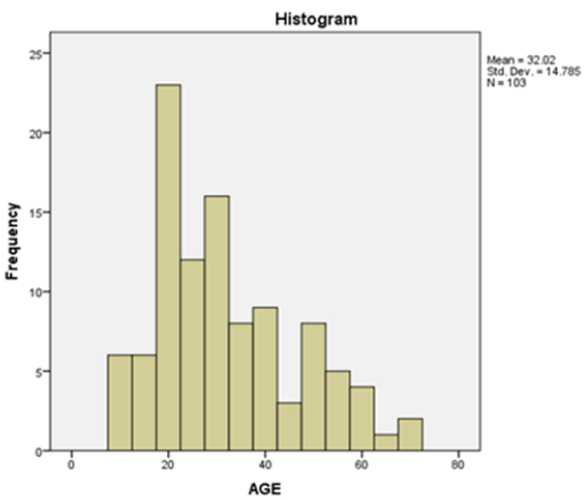

Figure 2 Age Distribution.

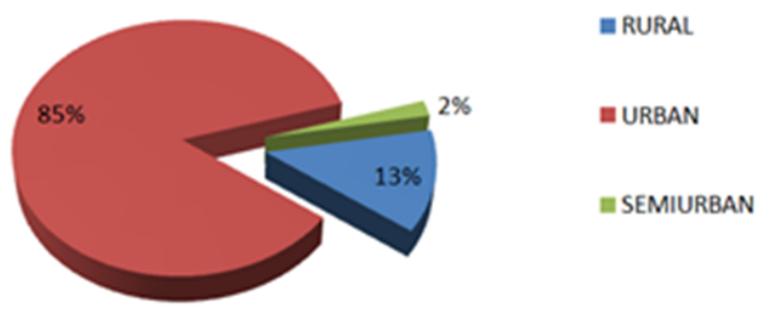

Figure 3 Rural Urban Distribution.

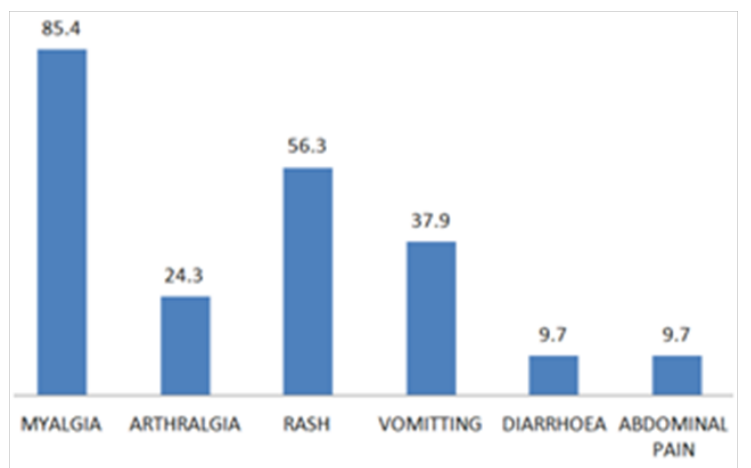

Figure 4 Presenting Symptoms.

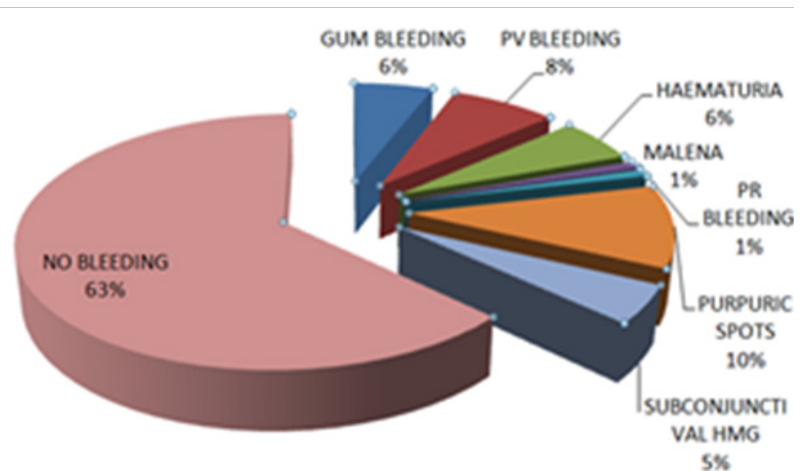

Figure 5 Bleeding Analysis.

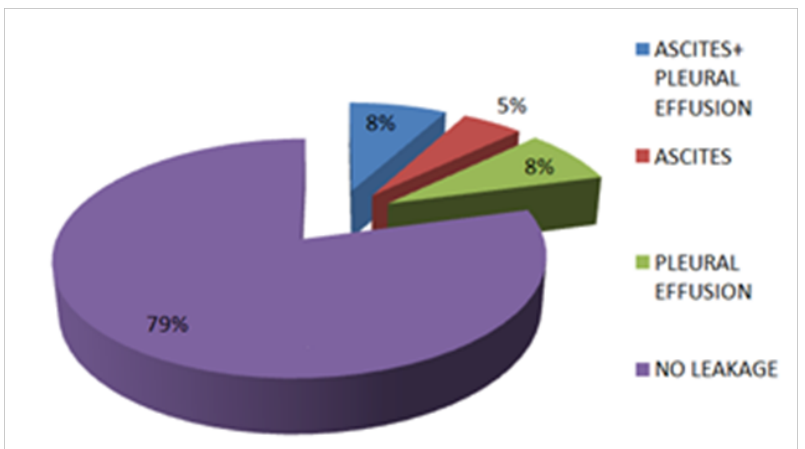

Figure 6 Analysis of Plasma Leakage.

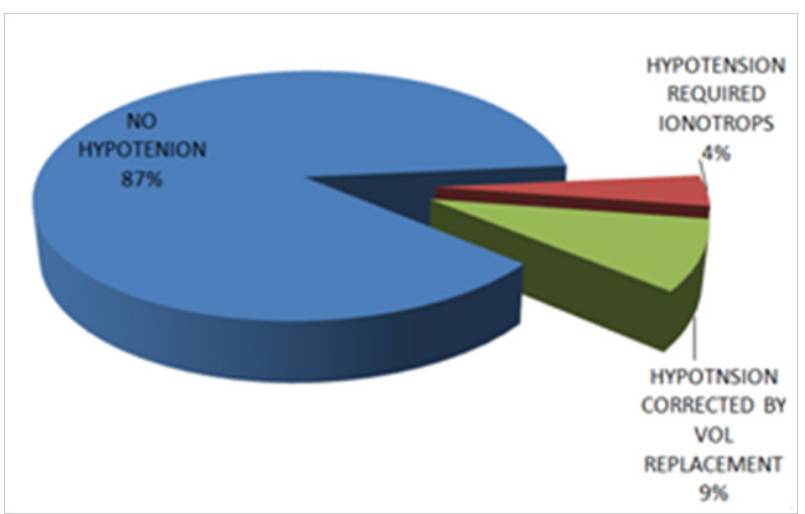

Figure 7 Hemodynamic Compromise and Management.

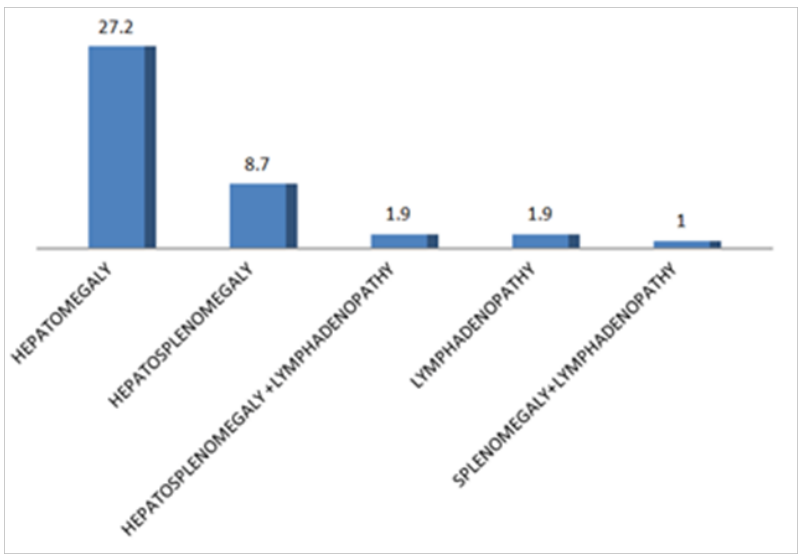

Figure 8 Organomegaly and Lyphadenopathy. 


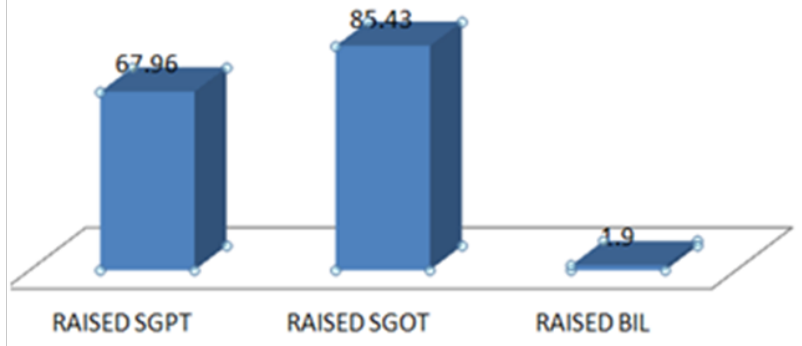

Figure 9 Liver Involvement I.

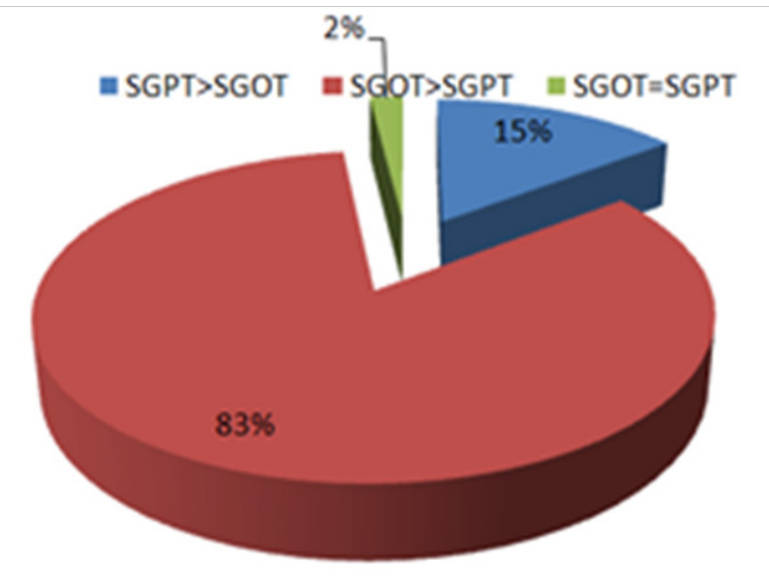

Figure 10 Liver Involvement 2.

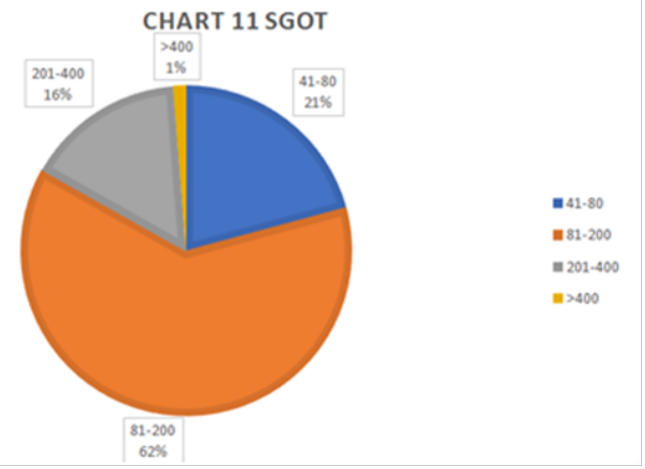

Figure I I SGOT.

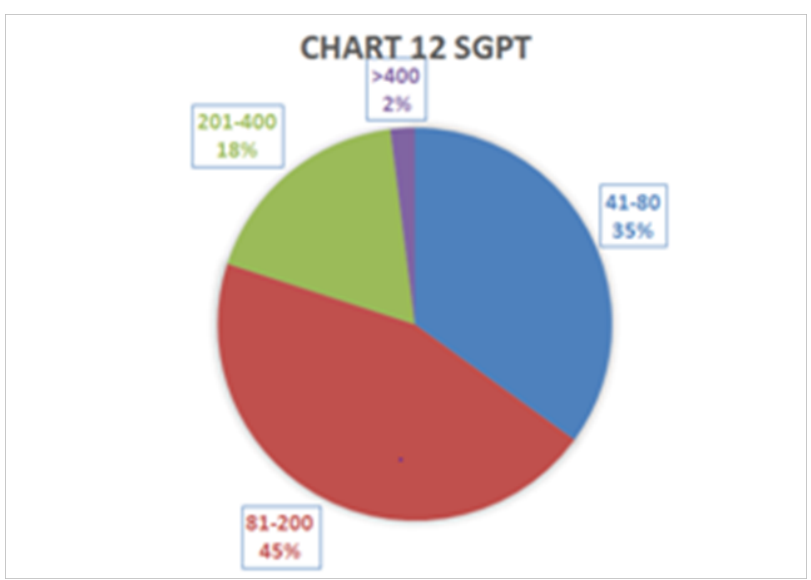

Figure I 2 SGPT.

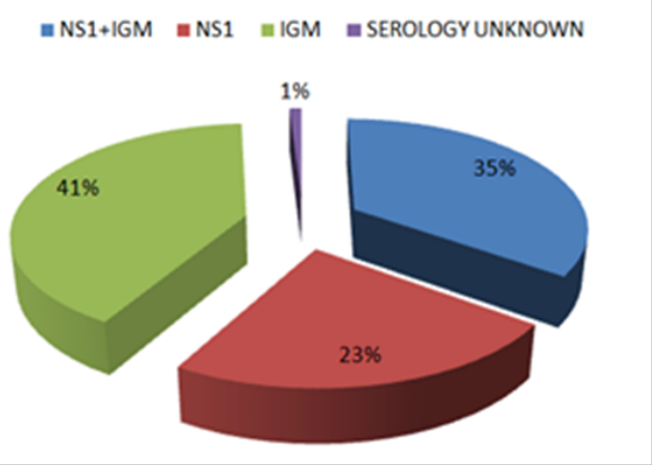

Figure 13 Serodiagnosis.
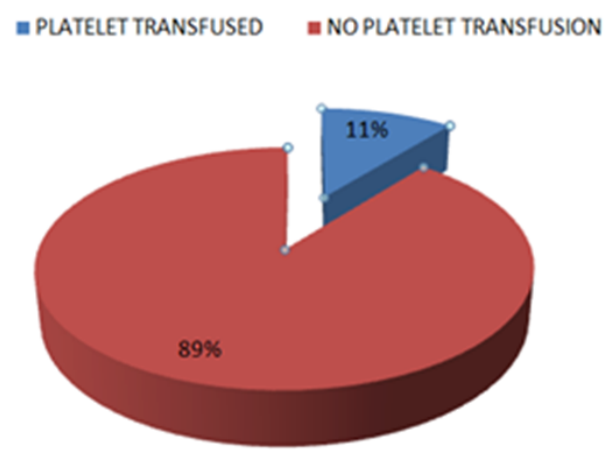

Figure 14 Platelet Transfusion.

\# PLATELET<10000 $=$ GUM BLEEDING $=$ PV BLEEDING $=$ EPISTAXIS

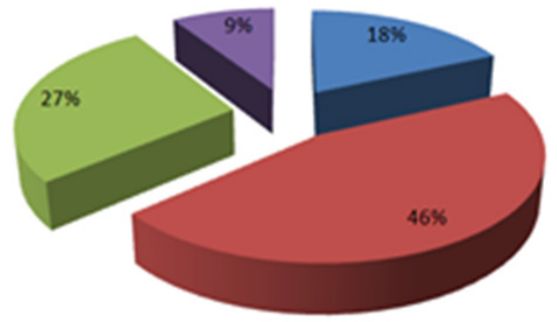

Figure 15 Indication of Platelet Transfusion.

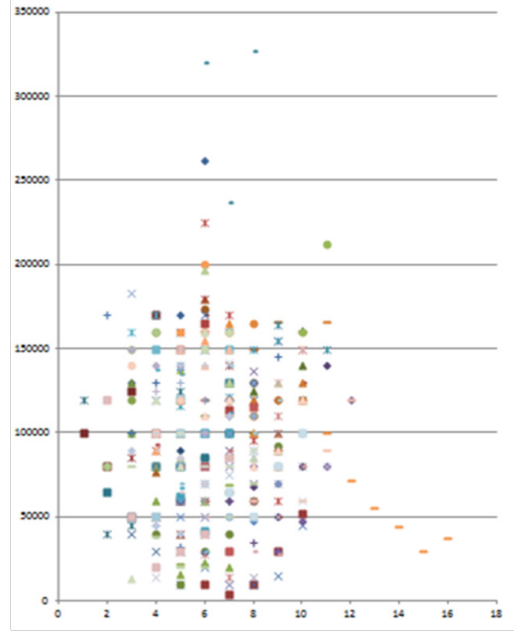

Figure 16 Platelet Count and Day of Fever. 


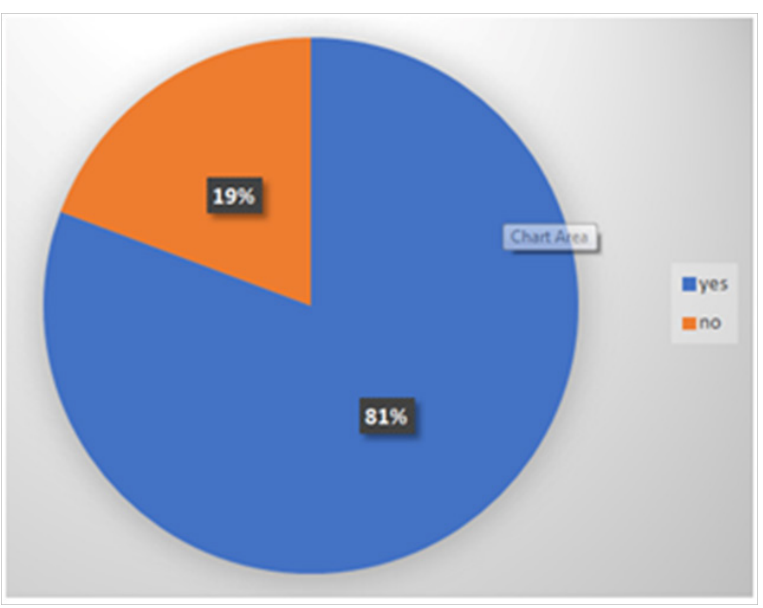

Figure 17 Pancreatic Involvement.

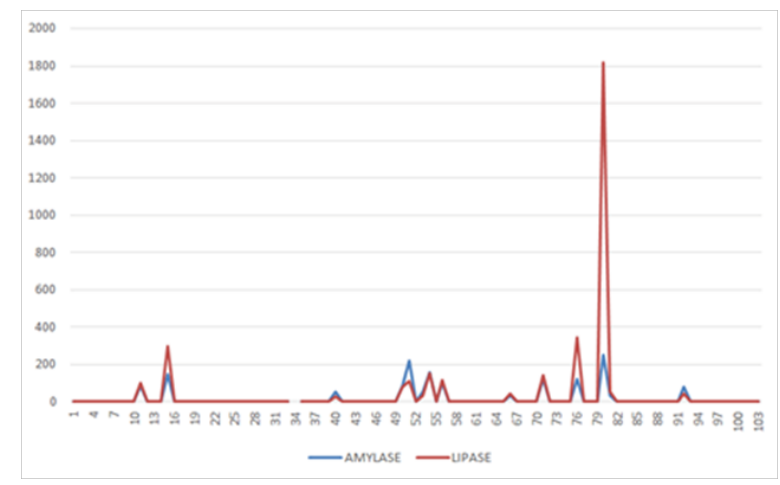

Figure 18 Pancreatic Involvement.

\section{Discussion}

Mandal et al. \& chatterjee et al. ${ }^{5,6}$ found that in dengue epidemic in the year 2012 most common manifestations were retro orbital pain, myalgia and amongst gastro intestinal manifestation abdominal pain, diarrhea and vomiting were present. In our study, total 103 dengue MAC ELISA positive or Dengue NS1Ag ELISA positive or both patients were included and analyzed. Amongst clinical manifestations most of the patients had myalgia $(85.4 \%)$, followed by rash $(56.3 \%)$ and vomiting (37.9\%). However only $24.3 \%$ of patients had arthralgia. But retro orbital pain was present only in $<1 \%$ patients. In our study, most more than half of the affected were male and most were from urban population with a mean age of 32 . Itha $\mathrm{S}$, et al. ${ }^{2}$ showed that dengue virus infection can cause significant liver injury in adults in the form of raised liver enzymes, hyperbilirubinemia, heaptomegaly, ascites. In this outbreak liver involvement was present in significant number of patients. In Liver function tests $85.43 \%$ patients had raised SGOT, $67.86 \%$ had raised SGPT and only minority (1.9\%) had raised bilirubin level. Amongst them liver enzymes were elevated and mostly SGOT elevation $>$ SGPT elevation was common for the disease. SGOT>SGPT were present in $86(83 \%)$ patients. $40 \%$ of patients had organomegaly amongst them majority had hepatomegaly. In analysis of SGOT elevation 2-5-fold elevation was common (62\%), 1 -2-fold elevation was only $16 \%$. But in analysis of SGPT elevation 2 -5-fold elevation was $45 \%$ and 1 -2-fold elevation was $35 \%$. This signifies more folds of increase in SGOT were specific for dengue fever in this outbreak. Plasma leakage was observed only in $21 \%$ of the patients amongst them pleural effusion was common. Bleeding diathesis is a feature of DF due to low platelet count and leakage from blood vessels. Bone marrow suppression, Immune mediated clearance, spontaneous aggregation of platelets to virus infected endothelium-all is responsible for such thrombocytopenia. Rachel et al. ${ }^{7}$ showed significant amount of thrombocytopenia and bleeding in dengue fever in a city of Kerala in the year 2003. In our study $37 \%$ of the patients had bleeding manifestations; amongst them purpuric spots were common followed by PV bleeding \& hematuria. We studied correlation between days counted from onset of fever and fall and rise of platelet count was noted accordingly; but no specific pattern could be noted. Chakravarti A et al. ${ }^{8}$ studied eight hundred ninety-three cases $(57.36 \%)$ were confirmed as serologically positive (NS1 Ag), out of which 199 (22.28\%) cases were positive for denguespecific IgM antibodies. Dengue NS1 Ag and Dengue IgM tests were done according to day of onset of fever as per NVBDCP guidelines. If the patient presented within 5 days of fever NS1 Ag was tested and irrespective of results Dengue $\operatorname{IgM}$ was tested at $5^{\text {th }}$ day of fever during treatment. But those who presented late (after $\geq 5$ days) only Dengue IgM was tested. In making a diagnosis $41 \%$ cases Dengue IgM was helpful followed by $35 \%$ for Dengue NS1Ag and only $1 / 5^{\text {th }}$ of patient had both tests reactive. Only $13 \%$ patient had hypotension of which majority was volume depleted. Chairulfatah A et al. ${ }^{9}$ showed that the patients with DHF/DSS in Bandung hospitals who received platelet transfusions, even if thrombocyte counts are above $25,000 / \mu \mathrm{l}$; platelet transfusions do not influence the incidence of severe bleeding. In our study platelet transfusion was needed in $11 \%$ of patients; in them nearly half were due to gum bleeding. Only $13.4 \%$ had pancreatic involvement and all of them had more lipase elevation than amylase. ${ }^{10,11}$

\section{Conclusion}

In the last decade, the world has witnessed different clinical presentations of the Dengue fever in different epidemics in different parts, even in the same regions and even with the period. Few atypical patterns in clinical, epidemiological and laboratory parameters are seen often. We found symptoms of dengue like myalgia, rash are common. We found significant hepatic involvement with SGOT $>$ SGPT and SGOT in more folds of elevation were typical pattern in dengue fever. Plasma leakage is having relatively low incidence and platelet count and day of fever has no specific association. Interestingly pancreatic involvement with high lipase than amylase seen in significant percentage $\left(1 / 10^{\text {th }}\right)$ of patients. These should always be kept in mind during an epidemic which would help in managing dengue fever. So, a sero-clinico-epidemiological surveillance and timely interventions are needed to identify the cases, so that its complications, outbreak morbidity and mortality can be minimized.

\section{Ethical approval}

We adhere to the ethics in publication as per guidelines of COPE.

\section{Acknowledgments}

None.

\section{Conflicts of interest}

The authors declare no conflicts of interest.

\section{Informed consent}

Informed consent was obtained from all individual participants included in the study. 


\section{References}

1. NVBDCP. National Guidelines for Clinical Management of Dengue Fever. National Vector Bourne Disease Control Programme, India, 2015. p. 1-37.

2. Itha S, Kashyap R, Krishnani N, et al. Profile of liverinvolvement in dengue virus infection. Natl Med J India. 2005;18(3):127-130.

3. Sasthya Bhavan. Glimpses of IDSP works West Bengal 2012. In:Disease surveillance Down to Earth Government of west Bengal. 2nd edn, Directorate of Health Services, Public Health Branch, Kolkata, India, 2013. p. 1-44.

4. Anandita Dawn. A Spatio-Temporal Analysis of Dengue Fever in West Bengal with Special Reference to Kolkata Municipal Corporation Area. IOSR Journal of Humanities. 2014;19(1):46-55.

5. Sanjay Kumar Mandal, Jacky Ganguly, Koelina Sil, et al. Clinical Profiles of Dengue Fever in a Teaching Hospital of Eastern India. Nati J Med Res. 2013;3(2):173-176.
6. Nandini Chatterjee, Kartik Patar, Mainak Mukhopadhyay, et al. An Observational Study of Dengue Fever in a Tertiary Care Hospital of Eastern India. Journal of the association of physicians of India. 2014;62:222-225.

7. Rachel Daniel, Rajamohananand Aby Zachariah Philip. A Study of Clinical Profile of Dengue Feverin Kollam, Kerala, India. Dengue Bulletin. 2005;29(1):194-195.

8. Chakravarti A, Kumaria R. Eco-epidemiological analysis of dengue infection during an outbreak of dengue fever, India. Virol J. 2005;2:32.

9. Chairulfatah A, Setiabudi D. Thrombocytopenia and platelet transfusions in DHF and DSS. Dengue Bulletin. 2003;27(1):138-143.

10. Sharma S, Sharma SK. Clinical profile of DHFin adults during 1996 outbreak in Delhi, India. Dengue Bulletin. 1998;22:20-27.

11. Lee IK, Liu JW, Yang KD. Clinical and laboratorycharacteristics and risk factors for fatality in elderly patients with dengue hemorrhagic fever. Am J Trop Med Hyg. 2008;79(2):149-153. 